\title{
Total Abdominal Hysterectomy with Bilateral Salpingo-Oophorectomy
}

National Cancer Institute

\section{Source}

National Cancer Institute. Total Abdominal Hysterectomy with Bilateral Salpingo-

Oophorectomy. NCI Thesaurus. Code C51761.

Surgical removal of all of the uterus, and the right and left fallopian tubes, and the right and left ovaries. 